\title{
Antimicrobial Activity of Silver Nanopartilcles in the Nanosilver Food Packages
}

\author{
Bi Hongmei $^{1 *}$, Zhang Xiaoqing ${ }^{2}$, Liang Ying ${ }^{1}$, Ding Dongxue ${ }^{1}$, Li Xue ${ }^{1}$, Wang Juntao ${ }^{1}$
}

${ }^{1}$ College of Science, Heilongjiang Bayi Agricultural University, Daqing 163319, PR China

${ }^{2}$ Department of Finance and Property, Qingbei Service Company of Daqing Oilfield, Daqing 163454, PR China

DOI: $10.36347 /$ sjavs.2021.v08i02.001

| Received: 18.01.2021 | Accepted: 01.02.2021 | Published: 03.02.2021

*Corresponding author: Bi Hongmei

\section{Abstract}

\section{Original Research Article}

Nowadays, nanosilver materials are widely used in the field of food packaging, which could significantly prolong the storage time of food. The migration of silver nanopartilcles in nanosilver packages is increasing continuously, while the case of the growth of Escherichia coli (E. coil) is not the same. In this paper, the antimicrobial activity to E. coil of silver nanoparticles is evaluated, which is useful to the evaluation of nanosilver food packages.

Key words: Food package, Silver nanoparticles, Escherichia coli (E. coil), Antimicrobial activity.

Copyright $\odot 2021$ The Author(s): This is an open-access article distributed under the terms of the Creative Commons Attribution 4.0 International License (CC BY-NC 4.0) which permits unrestricted use, distribution, and reproduction in any medium for non-commercial use provided the original author and source are credited.

\section{INTRODUCTION}

Nowadays, food packaging is not only packages of food; it could promote the quality of food to a certain extent. For this aim, the nanosilver food packages are competitive in this field. Silver-based material is a kind of antibiotic with a broad spectrum of activity, which have also attracted great interest owing to its photoactivity of semiconductor photocatalysis, antibacterial activity and the presence of nanocrystallites [1]. It is reported that the more the silver nanoparticles are, the better the antimicrobial activity would be.

The effects of silver nanoparticles on organisms and plants have been revealed in recent years $[2,3]$. Many studies have reported that the silver nanoparticles (AgNPs) do have influence on viruses and bacteria [4-6]. Ahmed fabricated olyquaternary phosphonium oligochitosans-AgNPs nanobiocomposites $\left(\mathrm{NBC}_{1,2}\right)$ to against hepatitis $\mathrm{A}$ virus (HAV), norovirus (NoV) and Coxsackievirus $\mathrm{B}_{4}$ $\left(\mathrm{Co}_{\mathrm{x}} \mathrm{B}_{4}\right)$ infections with great virucidal activities [4]. Sondi reported that silver nanoparticles could suppress the growth of Gram-negative bacteria and Grampositive bacteria [5]. Sandra used the mixture of the disinfectant $\mathrm{Ca}(\mathrm{OCl})_{2}$ and $\mathrm{AgNPs}$ against isolated bacteria, as well as inactivation of coliforms and pathogenic bacteria during percolation through bactericidal filter paper [6].
That is to say that a great amount of silver nanoparticles could do harm to animals. And too much intake into human body may have influence on the health of people, it is very important to detect the amount of silver nanoparticles in food packages [7], and it is useful to evaluate the antimicrobial to Escherichia coli (E. coil) of silver nanoparticles migrated from the nanosilver food packages.

\section{MATERIALS AND METHODS Materials and apparatus}

Four commercially available nanosilver/polypropylene milk storage packages were selected as research objects, and marked as A, B, C, D, respectively. Fresh milk of the same date was poured into the above four packages, and the packages were soaked for 30 days at room temperature. Every 5 days, milk samples were poured into petri dishes with violet red bile agar (VRBA).

Antimicrobial activity of silver nanoparticles by plate counting method

Because of the sensitivity of silver nanoparticles to E. coil, the milk samples were detected for the growth situation of E. coli by food microbiology detection with plate counting method.

\section{RESULTS AND DISCUSSION}

According to plate counting method, the milk samples were diluted to suitable concentration and mixed with VRBA on a superclean table. And then the 
milk samples were placed in constant temperature incubator for $24 \mathrm{~h}$. The number of E. coli was counted by digit auto-counter.

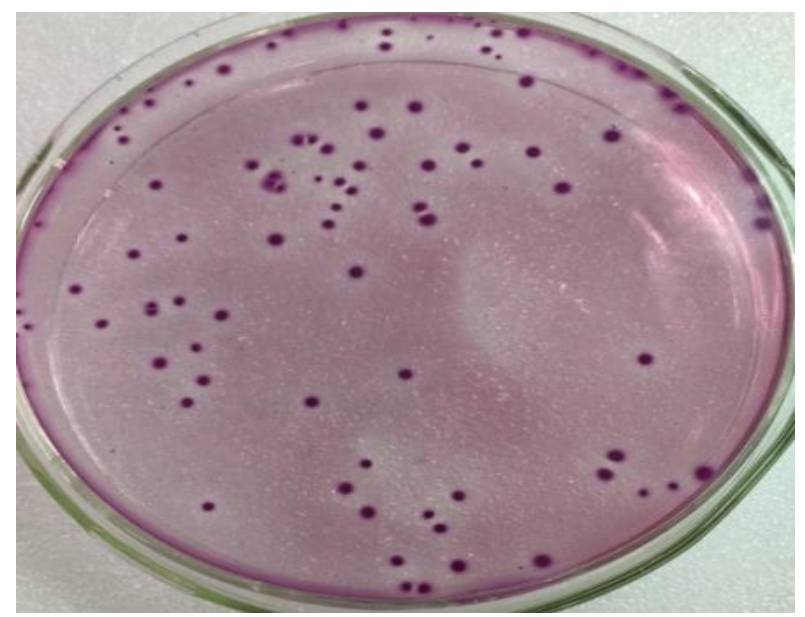

Fig-1: Typical and suspected Escherichia coli colonies on VRBA plates

The antimicrobial mechanism of silver nanoparticles on E. coli can be explained as follows: firstly, silver nanoparticles enter into cell walls, and have effect on cell membranes; secondly, when the concentration of silver nanoparticles reaches a certain amount, the DNA of bacteria lost the ability of copy, and E. coli could not metabolize, reproduce, and then die ultimately. While in the nanosilver packages, the migration of different samples of different brands were obviously different.

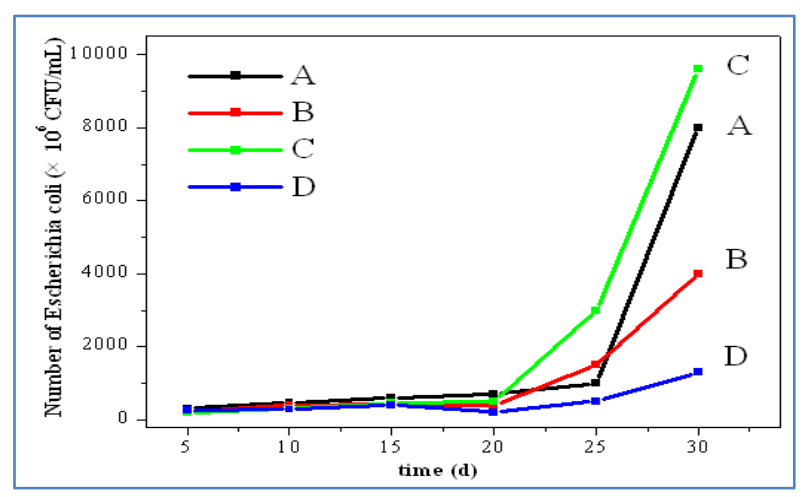

Fig-2: Number of Escherichia coli colonies of four nanosilver/polypropylene milk storage packages

In the four package samples, the growth situations of E. coli were similar; the number of E. coli colonies was obviously increasing after 20th day. It could be seen from the 4 brands of packages that the antimicrobial ability of silver nanoparticles behave excellent in the former 20 days, when it was longer than 20 days, the growth of E. coli was drastically increasing. The $\mathrm{D}$ band package behaved better. It didn't mean that milk could be stored in the nanosilver/polypropylene milk storage packages for more than 20 days, there were other indices to detect whether the milk had gone bad after all.

\section{CONCLUSION}

In this paper, the antimicrobial to E. coil of silver nanoparticles were evaluated here. The result showed that antimicrobial ability of the 4 brands of packages was better in the former 20 days, and the D brand package behaved much better. The reason was attributed to the different production technics of producing packages (includes polymer constituents, additive agents added, the particle diameter of silver nanoparticles etc).

\section{ACKNOWLEDGEMENTS}

This work was supported by Directive science and technology project of Daqing in China (Study on the migratory behavior of silver nanoparticles in food packages, 2020).

\section{REFERENCE}

1. Li Li, Chanjuan Zhao, Yadong Zhang, Jianfeng Yao, Wenjian Yang, Qiuhui Hu, Cailin Wang, Chongjiang Co. Effect of stable antimicrobia nanosilver packaging on inhibiting mildew and instorage of rice. Food Chemistry. 2017; 215:477482.

2. Liu R, Sun F, Zhang L. Evaluation on the toxicity of nano Ag to bovine serum albumin. Sci Total Environ. 2009; 407(13):4184-4188.

3. Chaoqun Wang, Xiao Liu, Zhen Han, Xu Zhang, Jingjing Wang, Kai Wang, Zhengtao Yang, Zhengkai Wei. Nanosilver induces the formation of neutrophil extracellular traps in mouse neutrophil granulocytes. Ecotoxicology and Environmental Safety. 2019; 183(15):109508.

4. Ahmed R. Sofy, Ahmed A. Hmed, Naglaa F. Abd El Haliem, Mohamed A.-E.Zein, Reda F. M. Elshaarawy. Polyphosphonium-oligochitosans decorated with nanosilver as new prospective inhibitors for common human enteric viruses. Carbohydrate Polymers. 2019; 226:115261.

5. Sondi I, Salopek-Sondi B. Silver nanoparticles as anti-microbial agent: A case study on E. coli as a model for Gram-negative bacteria. Journal of Colloid and Interface Science. 2004; 275(1):177182.

6. Asmaa N. Mohammed. Resistance of bacterial pathogens to calcium hypochlorite disinfectant and evaluation of the usability of treated filter paper impregnated with nanosilver composite for drinking water purification. Journal of Global Antimicrobial Resistance. 2019; 16:28-35.

7. Yang Yuhan, Zhang Xiaoqing, Li Hui, Wang He, Liu Ning, Qiu Shi, Bi Hongmei. Study on the Migration of Silver Nanoparticles from Nano silver Food Packages into Food Liquid [J]. Scholars Journal of Agriculture and Veterinary Sciences. 2021, 8(1): 4-7. 